\title{
Equation of state and transport processes in self-similar spheres
}

\author{
W. Barreto ${ }^{1,2}$, C. Peralta ${ }^{1}$ and L. Rosales ${ }^{3}$ \\ ${ }^{1}$ Laboratorio de Física Teórica, Departamento de Física, Escuela de Ciencias, Núcleo de Sucre, \\ Universidad de Oriente, Cumaná, Venezuela \\ ${ }^{2}$ Centro de Astrofísica Teórica, Universidad de los Andes, Mérida, Venezuela \\ 3 Sección de Física, UNEXPO Antonio José de Sucre, Puerto Ordaz, Venezuela
}

\begin{abstract}
We study the effect of transport processes (diffusion and free-streaming) on a collapsing spherically symmetric distribution of matter in a self-similar spacetime. A very simple solution shows interesting features when it is matched with the Vaidya exterior solution. In the mixed case (diffusion and freestreaming), we find a barotropic equation of state in the stationary regime. In the diffusion approximation the gravitational potential at the surface is always constant; if we perturb the stationary state, the system is very stable, recovering the barotropic equation of state as time progresses. In the free-streaming case the self-similar evolution is stationary but with a nonbarotropic equation of state.
\end{abstract}

PACS numbers: 04.40.-b, 04.40.Dg 


\section{INTRODUCTION}

The formation of compact objects is usually preceded by an epoch of radiative collapse [1]. One of the main difficulties in the study of these systems is that there is no reliable information about the equation of state in the central regions of superdense stars, such as neutron stars, and this leads to assumptions of a very general nature [2].

Few exact solutions to the Einstein equations are relevant to gravitational collapse. For this reason, new collapse solutions are very useful, even if they are simplified ones [3]. It is well known that the field equations admit homothetic motion [3-6]. Applications of homothetic similarity range from modeling black holes to producing counterexamples to the cosmic censorship conjecture [7-14].

The natural formulation of the collapse problem is that of initial values, with an a priori defined equation of state. However, there are only a few solutions to the Einstein field equations with a well defined equation of state. It has been shown that the only perfect fluid equation of state compatible with self-similarity is the barotropic one [4].

Emission of photons or neutrinos is a typical process in the evolution of massive stars. The only plausible mechanism to deliver almost all the binding gravitational energy, during the collapse toward a neutron star, is that of neutrino emission [15]. It seems clear that the free-streaming process is associated with the initial stages of the collapse, while the diffusion approximation becomes valid toward the final stages.

In this paper, we explore self-similar gravitational collapse. For such an assumption, we recast the geometrical variables so as to have an explicit radial dependence. The fluid is considered with heat flow (the diffusion approximation) or free propagation in the radial direction (free-streaming). The interior solution is matched to an exterior (Vaidya) solution by means of the Darmois-Lichnerowicz conditions.

We discuss neither the microprocesses that produce free-streaming, nor the temperature distribution during diffusion. For the latter another approach is necessary to avoid pathological behavior [16].

\section{THE FIELD EQUATIONS AND MATCHING}

For the geometric description of the interior of the distribution we use the radiation metric in the spherical Bondi form [17]

$$
d s^{2}=e^{2 \beta}\left(\frac{V}{r} d u^{2}+d u d r\right)-r^{2}\left(d \theta^{2}+\sin ^{2} \theta d \phi^{2}\right)
$$

where $\beta$ and $V$ are functions of $u$ and $r$. Here $u$ is a time-like coordinate, $r$ is a null coordinate $\left(g_{r r}=0\right)$, that is, $r \geq 0$ is an affine parameter along the null generators of $u=$ constant null hypersurfaces, and $\theta, \phi$ are the usual angular coordinates; we are using geometrized units $(c=G=1)$.

The hydrodynamic scenario, as viewed by a local Minkowskian observer comoving with the fluid (with velocity $-\omega$ ), consists of an isotropic fluid of density $\rho$ and pressure $p$,

unpolarized energy density $\epsilon$ and heat flux $q$ traveling in the radial direction. Therefore, for 
this comoving observer, the covariant energy-momentum tensor is

$$
\left(\begin{array}{cccc}
\rho+\epsilon & -q-\epsilon & 0 & 0 \\
-q-\epsilon & p+\epsilon & 0 & 0 \\
0 & 0 & p & 0 \\
0 & 0 & 0 & p
\end{array}\right)
$$

Note that the velocity of matter in the Bondi coordinates is

$$
\frac{d r}{d u}=\frac{V}{r} \frac{\omega}{1-\omega} .
$$

We can write the Einstein field equations as [18]

$$
\begin{gathered}
\frac{4 \pi r^{2}}{\left(1-\omega^{2}\right)}\left[\rho+p \omega^{2}+2 \omega q+\epsilon(1+\omega)^{2}\right]=\tilde{m}_{, r}-\frac{e^{-2 \beta} \tilde{m}_{, u}}{(1-2 \tilde{m} / r)}, \\
\frac{4 \pi r^{2}}{(1+\omega)}[(\rho-\omega p)-(1-\omega) q]=\tilde{m}_{, r}, \\
\frac{2 \pi r(1-\omega)(\rho+p-2 q)}{(1-2 \tilde{m} / r)(1+\omega)}=\beta_{, r}, \\
8 \pi p=-2 e^{-2 \beta} \beta_{, u r}+\left[3 \beta_{, r}\left(1-2 \tilde{m}_{, r}\right)-\tilde{m}_{, r r}\right] / r+(1-2 \tilde{m} / r)\left(2 \beta_{, r r}+4 \beta_{, r}^{2}-\beta_{, r} / r\right),
\end{gathered}
$$

where the comma subscript represents partial differentiation with respect to the indicated coordinate and $\tilde{m}$ is the Bondi mass defined by $\tilde{m}=(r-V \exp (-2 \beta)) / 2$.

The exterior space-time is described by the Vaidya radiating metric [19]. In order to match this to the interior solution, we use the Darmois-Lichnerowicz conditions. These are equivalent to the continuity of the functions $\beta$ and $\tilde{m}$ across the boundary of the sphere, and to the condition $[20,21]$

$$
\left[-\beta_{, u} e^{2 \beta}+(1-2 \tilde{m} / r) \beta_{, r}-\tilde{m}_{, r} /(2 r)\right]_{a}=0
$$

which is equivalent to $p_{a}=q_{a}$. The subscript $a$ indicates that the quantity is being evaluated at the surface $r=a(u)$.

\section{SPHERICAL AND SELF-SIMILAR INTERIOR SOLUTIONS}

Self-similarity is invariably defined by the existence of a homothetic Killing vector field [4]. A homothetic vector field on the manifold is one that satisfies $£_{\xi} \mathbf{g}=2 n \mathbf{g}$ on a local chart, where $n$ is a constant on the manifold and $£$ denotes the Lie derivative operator. If $n \neq 0$ we have a proper homothetic vector field and it can always be scaled to have $n=1$; if $n=0$ then $\xi$ is a Killing vector on the manifold [22-24]. So, for a constant rescaling, $\xi$ satisfies $£_{\xi} \mathbf{g}=2 \mathbf{g}$ and has the form $\xi=\Lambda(u, r) \partial_{u}+\lambda(u, r) \partial_{r}$. If the matter field is a perfect 
fluid, the only equation of state consistent with $£_{\xi} \mathbf{g}=2 \mathbf{g}$ is a barotropic one [4]. The homothetic equations reduce to $\xi(X)=0, \xi(Y)=0, \lambda=r$ and $\Lambda=\Lambda(u)$, where $X \equiv \tilde{m} / r$ and $Y \equiv \Lambda e^{2 \beta} / r$. Therefore, $X=X(\zeta)$ and $Y=Y(\zeta)$ are solutions if the self-similar variable is defined as $\zeta \equiv r \exp \left(-\int d u / \Lambda\right)$. Here we assume that $X=C_{1} \zeta^{k}$ and that $Y=C_{2} \zeta^{l}$, where $C_{1}, C_{2}, k$ and $l$ are constants. This power-law dependence on $\zeta$ is based on the fact that any function of $\zeta$ is solution of $£_{\xi} \mathbf{g}=2 \mathrm{~g}$. As we shall see, this simplifying assumption is not devoid of physical meaning. Demanding continuity of the first fundamental form we get the following metric solutions:

$$
\begin{gathered}
\tilde{m}=\tilde{m}_{a}(r / a)^{k+1}, \\
e^{2 \beta}=(r / a)^{l+1} .
\end{gathered}
$$

Condition (7) then implies that the local radial velocity $\omega$ is determined at the surface in terms of the gravitational potential $\tilde{m}_{a} / a$ and the parameters $k$ and $l$ :

$$
\omega_{a}=1-\frac{\left(1-2 \tilde{m}_{a} / a\right)}{\tilde{m}_{a} / a} \frac{(1+l)}{(1+k)} .
$$

Equations (2) and (3) evaluated at the surface constitute the system of (ordinary differential) equations in $a(u)$ and $\tilde{m}_{a}(u)$, to be integrated while taking into account Eq. (10). Thus, the dynamics at the surface is completely determined if we establish how energy is exchanged with the exterior. In fact, we have found that self-similarity determines the luminosity profiles $[25,26]$.

\section{TRANSPORT PROCESSES}

In order to explore the effect of the transport processes on self-similar gravitational collapse, we consider below a combination of the diffusion and free-streaming mechanisms, and the action of each one separately. In particular we discuss whether the barotropic equation of state holds in each case.

\section{A. Mixed}

If the transport mechanism is mixed, we require additional information. Only in this case do we suppose orthogonality between the four-velocity and the homothetic vector. This condition has been employed to obtain static solutions [27] and it also establishes a relationship between the homothetic vector and the equation of state [28]. Another meaning attributable to the orthogonality condition is that the group, generated by the homothetic vector, is acting upon the three-space comoving with the observer. The referred condition, together with $£_{\xi} \mathbf{g}=2 \mathbf{g}$, can thus be seen as a covariant definition of self-similarity [29]. Therefore, from the orthogonality condition we obtain the temporal component of the homothetic vector:

$$
\Lambda=\tilde{m}_{a}(1+l) /(1+k)
$$


The radiation flux at the surface is then determined from the symmetry equations. The heat flux at the surface is also determined from equation (6) or, equivalently, from $T_{1 ; \mu}^{\mu}=0$ evaluated at the surface. Now, feeding back (10) and (11) into the symmetry equations, we find algebraically by means of symbolic manipulation with REDUCE, two restrictions which allow us to satisfy the symmetry equations: (i) $k=-l$; (ii) $k=\left[\left(1-2 \tilde{m}_{a} / a\right)(l+2)-\right.$ $1] /\left(2 \tilde{m}_{a} / a\right)$. Restriction (ii) leads to $\omega_{a}=-1$ (through Eq. (10)), that is, the fluid collapses at light speed; we must reject this possibility. Under restriction (i), $k=-l$, the homothetic symmetry is preserved at all points of the space-time, when we integrate numerically (using the Runge-Kutta method) the system of equations at the surface. Specifically, for $k=0$ the physical variables are well behaved and the equation of state is barotropic in the whole sphere; that is, the ratio $p / \rho$ is constant at all points of the material (see Figure 1). All shells collapse with the emission of energy. The pressure, density, heat flow and radiation flux are stationary inside the sphere. The matter velocity decreases toward the center and is constant at the surface. Observe that $p \approx(3 / 5) \rho$, as we expect, because the heat flow diminish the effective gravitation or, in others words, the equation of state is softened by the diffusive process [30,18]. For $k \neq 0$ the equation of state is not longer barotropic and the physical variables are not stationary, although the interior space-time is self-similar.

\section{B. Diffusion}

In the diffusion approximation $(\epsilon=0)$ we again obtain the heat flux at the surface from equation (6) or $T_{1 ; \mu}^{\mu}=0$ evaluated at the surface. From the symmetry equations evaluated at the surface, we deduce that the gravitational potential at the surface, $\tilde{m}_{a} / a$, is a function of only $k$ and $l$. The surface equations thus reduce to one differential equation for $a$ (or $\tilde{m}_{a}$ ). This situation leads us to four possible restrictions: (i) $k=-l$; (ii) $l=-1$ which is equivalent to $\omega_{a}=1$ (see Eq. (10)), that is, the fluid explodes at light speed; (iii) a complicated polynomial of degree three in $k$ with coefficients depending on $l$; (iv) a complicated polynomial of degree seven in $k$ with coefficients depending on $l$. We solved analitycally the polynomial of degree three (using REDUCE), obtaining one real and two complex solutions. All these must be rejected because they do not have physical meaning for a wide interval of numerical values of $l$. After solving numerically the polynomial of degree seven, we found that the physically acceptable models are similar to those that emerge from restriction (i), $k=-l$. In any case, the gravitational potential at the surface is constant $\left(\tilde{m}_{a} / a\right.$ depending only on $k$ ) although the distribution collapses. If we perturb this stationary state, we find numerically that the system recovers the barotropic equation of state only for $k=0$ (see Figure 2). Perturbations consist of enhancing or diminishing the gravitational potential at the surface, while preserving the values of $k$. Therefore, we must integrate numerically (using the Runge-Kutta method again) the two perturbed differential equations at the surface. It is important to stress that the symmetry equations are satisfied everywhere and at all times when we perturb the system. 


\section{Free-streaming}

In this transport process $(q=0)$ the boundary condition reduces to $p_{a}=0$. We obtain again a gravitational potential which is constant at the surface (from $T_{1 ; \mu}^{\mu}$ evaluated at the surface). Therefore, the luminosity profile and the temporal component of the homothetic vector are determined from one of the symmetry equations. No restrictions appear on the parameters $k$ and $l$, and no barotropic behavior is found, at least for the cases examined.

\section{CONCLUSION}

We have studied the effect of diffusion and free-streaming on self-similar gravitational collapse using the solutions to the Einstein equations given by (8) and (9). We find that the equation of state is barotropic only when diffusion occurs and the parameter $k$ is zero. In general $(k \neq 0)$ the equation of state is not barotropic, although self-similarity holds everywhere. Thus for non-perfect fluids self-similarity is connected with a barotropic equation of state only under special conditions. The heat flow seems to be crucial for this result. Viscosity in self-similar distributions has also been considered previously and the same result emerges; that is, the equation of state can be barotropic [26]. We have found relativistic and self-similar examples compatible with a nonbarotropic equation of state [31], at least within our chosen simple solutions and the orthogonality condition between the generator of the group and the four-velocity. It would be of interest to attempt to relax some of our suppositions in order to investigate how general our conclusions are.

\section{ACKNOWLEDGMENTS}

We benefited from research support by the Consejo de Investigación under Grant CI-51001-0774/96 of the Universidad de Oriente. We would like to thank Loren Lockwood for his helpful reading and valuable comments. 


\section{REFERENCES}

[1] F. Fayos, X. Jaén, E. Llanta and J. Senovilla, Phys. Rev. D 45, 2732 (1992).

[2] R. Tikekar, J. Math. Phys. 31, 2454 (1990).

[3] A. Ori and T. Piran, Phys. Rev. D 42, 1068 (1990).

[4] M. E. Cahill and A. H. Taub, Commun. Math. Phys. 21, 1 (1971).

[5] R. N. Henriksen and K. Patel, Gen. Rel. Grav. 23, 527 (1991).

[6] K. Lake and T. Zannias, Phys. Rev. D 41, 3866 (1990).

[7] J. Carr and S. Hawking, MNRAS 168, 399 (1974).

[8] G. V. Bicknell and R. N. Henriksen, Ap. J. 219, 1043 (1978).

[9] G. V. Bicknell and R. N. Henriksen, Ap. J. 225, 237 (1978).

[10] J. Carr and A. Yahil, Ap. J. 360, 330 (1990).

[11] D. M. Eardley, J. Isenberg, J. Marsden and V. Moncrief, Comm. Math. Phys. 106, 137 (1986).

[12] K. Lake, Phys. Rev. Lett. 68, 3129 (1992).

[13] P. R. Brady, Phys. Rev. D 51, 4168 (1995).

[14] B. J. Carr and A. A. Coley, gr-qc/9806048.

[15] L. Herrera, Campos gravitacionales en la materia: la otra cara de la moneda. "II Escuela Venezolana de Relatividad, Campos y Astrofísica". Facultad de Ciencias, Universidad de los Andes, Mérida, Venezuela (1996).

[16] J. Martínez, Phys. Rev. D 53, 6921 (1996).

[17] H. Bondi, Proc. Roy. Soc. London A 281, 39 (1964).

[18] W. Barreto and L. Núñez, Ap. Sp. Sc. 178, 261 (1991).

[19] P. C. Vaydia, Phys. Rev. 83, 10 (1951).

[20] L. Herrera and J. Jiménez, Phys. Rev. D 28, 2987 (1983).

[21] L. Herrera, J. Jiménez, and G. Ruggeri, Phys. Rev. D 22, 2305 (1980).

[22] G. S. Hall, Gen. Rel. Grav. 20, 671 (1988).

[23] G. S. Hall, J. Math. Phys. 31, 1198 (1990).

[24] J. Carot, L. Mas and A. M. Sintes, J. Math. Phys. 35, 3560 (1994).

[25] W. Barreto and L. Castillo, J. Math. Phys. 36, 5789 (1995).

[26] W. Barreto, J. Ovalle and B. Rodríguez, Gen. Rel. Grav. 30, 15 (1998).

[27] L. Herrera, J. Jiménez, L. Leal, J. Ponce de León, M. Esculpi and V. Galina, J. Math. Phys. 25, 3274 (1984).

[28] C. Kolassis and J. B. Griffiths, Gen. Rel. Grav. 28, 805 (1996).

[29] L. Herrera (private communication).

[30] W. Barreto, L. Herrera and N. Santos, Ap. J. 344, 158 (1989).

[31] D. Pollney and T. Zannias, Phys. Rev. D 56, 8086 (1997). 


\section{FIGURES}
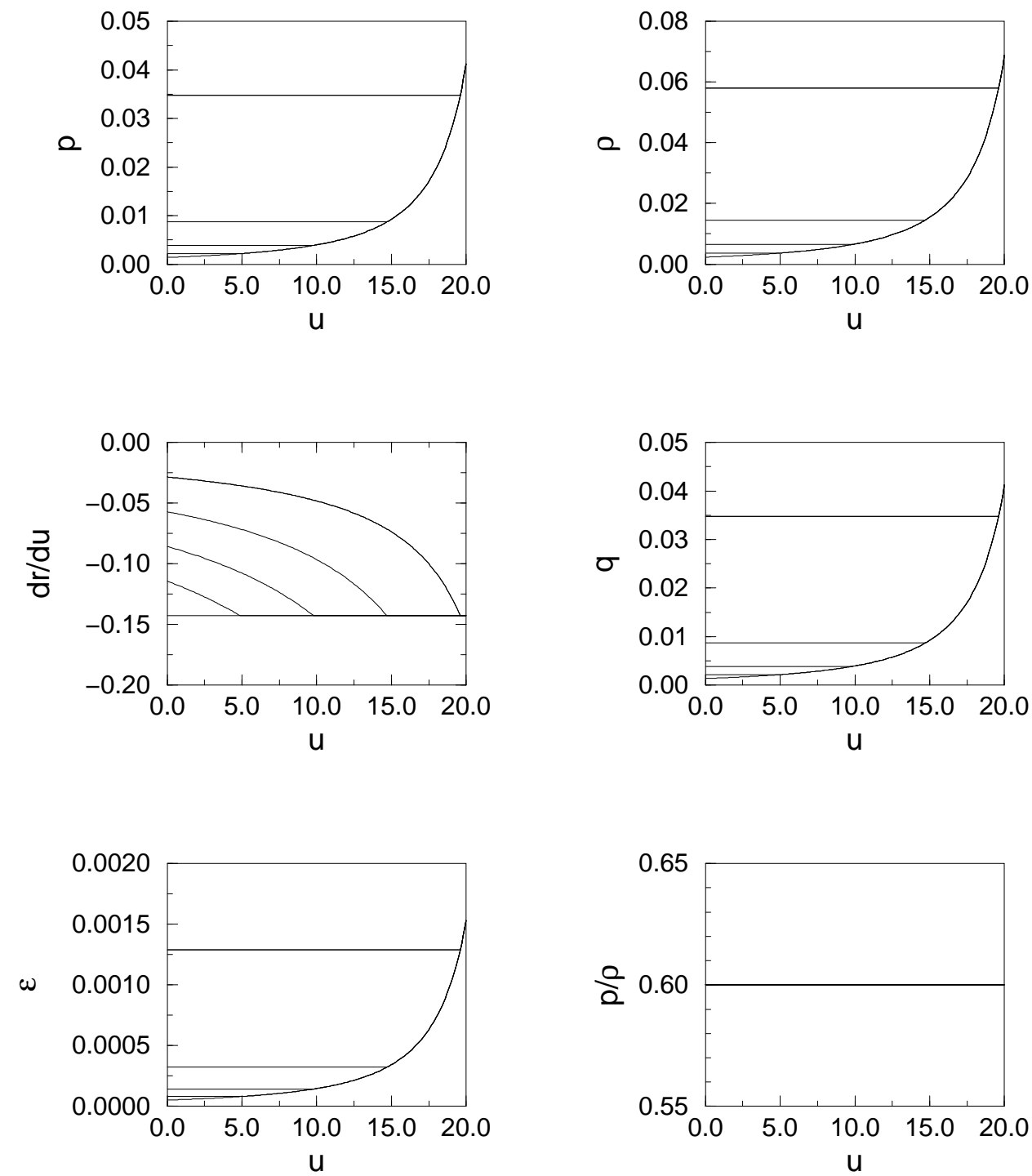

FIG. 1. Evolution of the physical variables for the mixed transport process with $k=0$. In each graph the curves correspond (from the uppermost to the lowermost) to ratios: $r / a(0)=0.2,0.4$, $0.6,0.8$ and 1.0, respectively. The ratio $p / \rho$ is the same at any point of the material. 


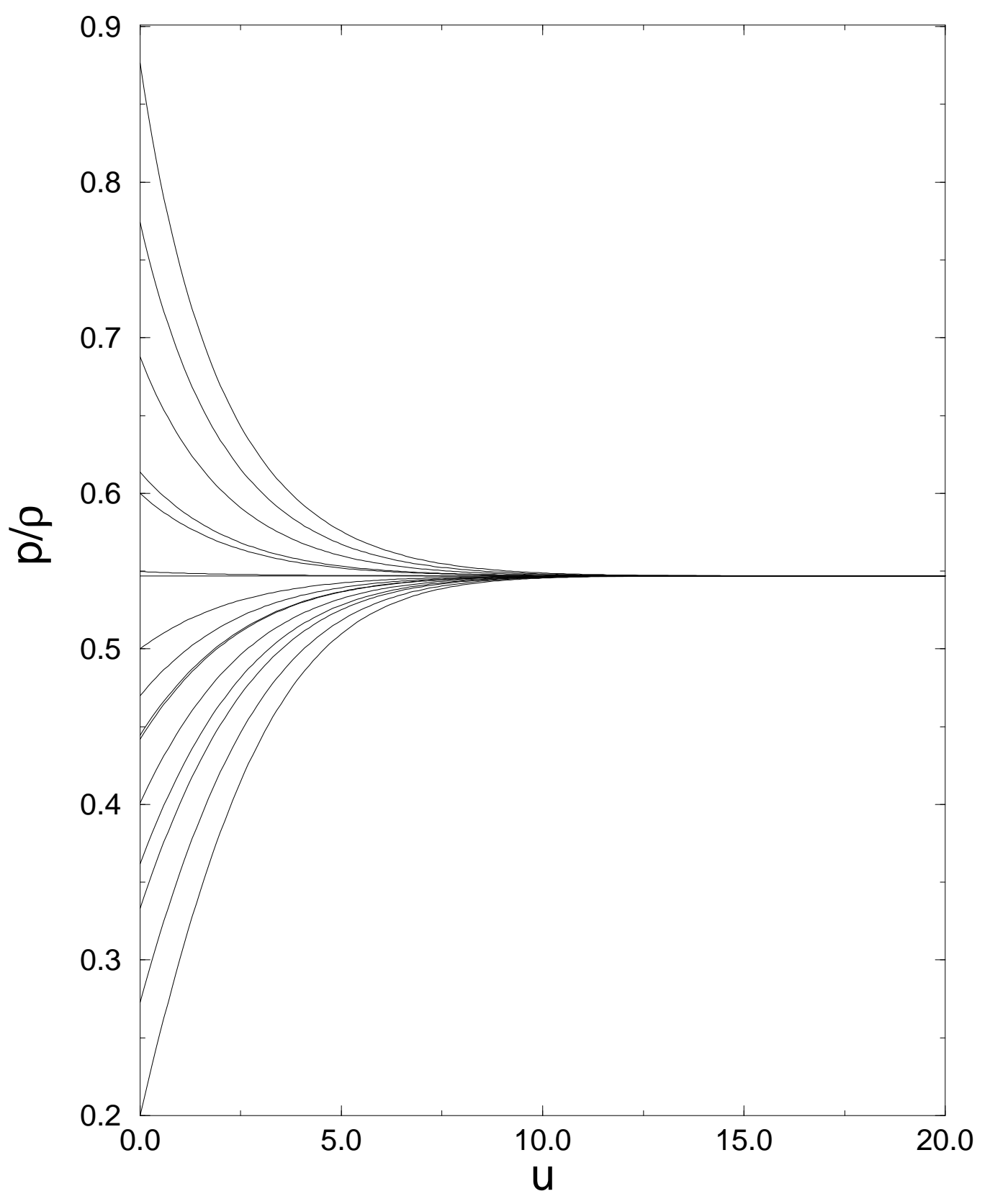

FIG. 2. The ratio $p / \rho$ as a function of retarded time in the diffusion approximation with $k=0$, considering perturbations of the stationary regime. 